\title{
Fault Diagnosis in Transformer Based on Weighted Degree of Grey Slope Incidence of Optimized Entropy
}

\author{
Anping Zhang, Sujie Geng \\ School of Economics and Management, Nanjing University of Science and Technology, Nanjing, Jiangsu Province, 210094, China
}

\begin{abstract}
Dissolved gas analysis (DGA) is an important method to find the hidden or incipient insulation faults of oil-immersed power transformer. However, code deficiency exists in the gas ratio methods specified by the IEC standard and complexity of fault diagnosis for power transformer. Hence a new model based on optimized weighted degree of grey slope incidence was put forward. Firstly, the entropy weight is used to determine objective weight of indices; then the model fault types are obtained by weighted degree of grey slope incidence. The combination of entropy weight with grey slope incidence analysis can fully utilize over all information of DGA and give full play to the superiority of grey slope incidence, which overcomes shortcomings of original grey slope incidence analysis. The experimental results also demonstrate that the improved method has higher accuracy compared with three-ratio method and general grey slope incidence analysis method. The diagnosis accuracy is $92.8 \%$.
\end{abstract}

\section{Introduction}

Electric transformer is not only the most important and most expensive equipment, but also one of the most accident-prone equipments in electric system. In order to ensure safety and credibility of the transformer, it is important to find out the potential fault in the transformer as soon as possible and forecast the development trend of fault. It is an important instrumentality to monitor the running of electric transformer and ensure its safe and economical running. Its running reliability has direct relation to the safe running of electric system.

At present, dissolved gas analysis (DGA) is still the extensively using method in transformer fault diagnosis [1-2]. For example, IEC three-ratio method has not enough codes to effectively troubleshoot a transformer's operational condition in practical use. In recent years, many scholars both at home and abroad have done a lot of research in the field of transformer fault diagnosis. They also put forward a lot of methods, such as combining dissolved gas analysis with fuzzy logic, rough set theory, Bayesian networks, artificial neural networks, artificial immune, new radial basis function networks, genetic algorithm and support vector machine. These methods are relatively effective for transformer fault diagnosis, but each has advantages and disadvantages. Such as artificial neural networks with self-organizing, adaptive, fault-tolerant, self-learning and highly nonlinear approximation capability, is a strong tool to process nonlinear system. But the accuracy of the diagnosis depends from the completeness of samples and it is unable to process the incomplete sample [3-4]. Fuzzy set theory is good at treating uncertainty knowledge, but the determination of fuzzy membership function requires expert experience or repeated test [5]. Support vector machine has a powerful generalization ability, and overcomes shortcomings like excessively fits, slow convergence and easy to fall into local extreme value exiting in neural networks. But training time is long, and repeating training samples are large. In addition, the classification capability is not satisfactory under the incomplete information [6-7].

Transformer fault system is a grey system which partial information is known and partial information is unknown, the essence of transformer fault diagnosis would be the whitening process of grey system [8-11]. Therefore, we can take DGA as the characteristic vector, and use the grey incidence analysis method to diagnose transformer fault. Since the incidence coefficients of general grey slope incidence analysis method fluctuate easily, combining with the analysis of grey sequence entropy, this paper presents the model based on weighted degree of grey slope incidence of optimized entropy [12]. And then we have proved the effectiveness and practical value of this improved method according to specific fault examples.

\section{The model of improved grey incidence analysis}

\subsection{The model of general grey slope incidence}

The basic idea of grey incidence analysis is judging 
whether a series of curves of sequences are close or not according to their correlation degree. Two curves are much closer, the correlation of sequences is bigger, contrarily, become more smaller. Therefore, the degree of grey incidence evaluates the correlation condition of two sequences on the whole. There is both a positive correlation and a negative correlation between them whose correlation degree can be reflected by the change in slope of sequences curves [13]. The definition of grey slope incidence can be summarized as follows.

Definition 1:

Supposing $X_{0}=\left[x_{0}(1), x_{0}(2), \ldots, x_{0}(n)\right]$ be a data sequence of a system's characteristic behavior, and $X_{i}=\left[x_{i}(1), x_{i}(2), \ldots, x_{i}(n)\right], i=1,2, \cdots, m \quad$ relevant factor sequences. And

$$
\begin{aligned}
& \Delta_{0}(k)=x_{0}(k)-x_{0}(k-1), k=2,3, \cdots, n \\
& \Delta_{i}(k)=x_{i}(k)-x_{i}(k-1), k=2,3, \cdots, n \\
& R_{i}=\max _{k} x_{i}(k)-\min _{k} x_{i}(k) \\
& R_{0}=\max _{k} x_{0}(k)-\min _{k} x_{0}(k) \\
& \gamma_{0 i}(k)=\operatorname{sgn}_{k} \frac{1}{1+\left|\frac{\left|\Delta_{i}(k)\right|}{R_{i}}-\frac{\left|\Delta_{0}(k)\right|}{R_{0}}\right|}, \\
& k=2,3, \cdots, n, i=1,2, \cdots, m
\end{aligned}
$$

where $\operatorname{sgn}_{k}=\left\{\begin{array}{cl}1, & \text { if } \Delta_{0}(k) \Delta_{i}(k) \geq 0 \\ -1, & \text { if } \Delta_{0}(k) \Delta_{i}(k) \prec 0\end{array}\right.$ denotes a incidence sign function, the $\gamma_{0 i}(k)$ represents the grey slope incidence coefficient. Then

$$
\gamma_{0 i}=\frac{1}{n-1} \sum_{k=2}^{n} \gamma_{0 i}(k)
$$

is referred to as the degree of grey slope incidence between $X_{0}$ and $X_{i}$.

\subsection{The model based on weighted degree of grey slope incidence of optimized entropy}

When calculating the grey slope incidence, we can't accurately reflect the relations between various sequences if we take the mean of slope incidence coefficient as grey slope incidence. Because the grey slope incidence coefficients of various points have a different influence on the data sequence of a system's characteristic behavior [14]. Accordingly, maybe it's better to add a weight to the model of calculating the grey slope incidence. The definition of weighted degree of grey slope incidence is as follows.

Definition 2: Given the data sequences:

$$
\begin{gathered}
X_{0}=\left[x_{0}(1), x_{0}(2), \ldots, x_{0}(n)\right] \\
X_{1}=\left[x_{1}(1), x_{1}(2), \ldots, x_{1}(n)\right] \\
\vdots \\
X_{i}=\left[x_{i}(1), x_{i}(2), \ldots, x_{i}(n)\right] \\
\vdots \\
X_{m}=\left[x_{m}(1), x_{m}(2), \ldots, x_{m}(n)\right]
\end{gathered}
$$

where $X_{0}$ is a data sequence of a system's characteristic behavior and $X_{i}$ is relevant factor sequences. Then,

$$
\gamma_{0 i}=\sum_{k=2}^{n} w_{k} \gamma_{0 i}(k)
$$

where $\gamma_{0 i}(k)$ represents the slope incidence coefficient between $X_{0}$ and $X_{i}$ at point $\mathrm{k}, \gamma_{0 i}$ is referred to as a weighted degree of grey slope incidence between $X_{0}$ and $X_{i}, w_{k}$ is known as the weight coefficient, and $\sum_{k=2}^{n} w_{k}=1\left(0 \prec w_{k} \prec 1\right)$ is required.

Obviously, $\gamma_{0 i}$ meets four axioms of grey incidence, namely normalization, even symmetry, globality and proximity.

The key to the model of weighted degree of grey slope incidence may be how to fix the weight values which can reflect the effect of each point's slope on whole grey incidence. We hope to keep these effects balanced and objective, so entropy weight method is applied to weighting the slope incidence coefficient.

Based on the assumption that the different elements relations are stable in the grey system, we will analyze the weighted slope incidence coefficient according to the grey entropy theory presented in paper [12]. First, this paper constructs a grey sequence based on the weighted slope incidence coefficient, and then it establishes an optimized model of the weight of slope incidence coefficients.

Definition 3: Let Map: $R_{j} \rightarrow P_{j}$

$$
p_{k}=\frac{w_{k} \gamma_{0 i}(k)}{\sum_{k=2}^{n} w_{k} \gamma_{0 i}(k)}
$$

be the distributed map of weighted slope incidence coefficient, map $p_{k}$ is referred to as the density value of weighted slope incidence coefficient distribution.

The information contained in this sequence $\left\{p_{k}\right\}$ can't be completely certain, and then, for any $\mathrm{k}$, there exits $p_{k} \geq 0$ and $\sum_{k=2}^{n} p_{k}=1$. According to the definition taken from paper [12], the sequence $\left\{p_{k}\right\}$ is a grey sequence. 
Definition 4: The given function

$$
H_{\otimes}\left(R_{i}\right)=-\sum_{k=2}^{n} p_{k} \ln p_{k}
$$

is the weighted grey incidence entropy of $X_{i}$.

The weighted grey incidence entropy $H_{\otimes}\left(R_{i}\right)$ share the same structure with Shannon entropy. They both have symmetry, non negativity, additive, convexity and extremum. However, Shannon entropy is probability entropy while grey entropy is non-probabilistic entropy, the grey properties of grey entropy depend on grey series' properties. However, according to the convexity and extremum of grey entropy function, this distinction does not affect the extremum properties of grey entropy.

As this sequence $\left\{p_{k}\right\}$ is grey sequence, on the basis of entropy increase principle of grey series presented in paper [12], the theorem of weighted slope incidence entropy increase can be given as follows.

Theorem 1: Assume that the distribution density sequence of weighted grey incidence coefficient is $P=\left\{p_{k} \mid \forall k, p_{k} \geq 0, \sum_{k=2}^{n} p_{k}=1\right\}$, and $H_{\otimes}\left(R_{i}\right)$ is referred to as incidence entropy, then any change that tends to equalize $p_{k}$ will increase incidence entropy $H_{\otimes}\left(R_{i}\right)$.

In the grey incidence analysis, the grey slope incidence coefficient reflects the influence of each point's slope on the data sequence of system's characteristic behavior. The influence ought to remain stable, so the incidence coefficient of slope at each point which is weighted should keep balance as much as possible [15-16]. Our goal is fixing the weight coefficient $w_{k}$ so that the weighted slope incidence coefficient distribution density value $p_{k}$ tends to balance. Therefore, on the basis of theorem 1, we exert a maximum constraint to the weighted incidence entropy $H_{\otimes}\left(R_{i}\right)$.

$$
\begin{aligned}
& \max _{\otimes} R_{i} \Rightarrow-\sum_{k=2}^{n} p_{k} \quad p \mathrm{n}_{k} \\
& \text { s.t } \sum_{k=2}^{n} w_{k}=1 w_{k} \geq \otimes ; \cdot 2, n,
\end{aligned}
$$

where

$$
p_{k}=\frac{w_{k} \gamma_{0 i}(k)}{\sum_{k=2}^{n} w_{k} \gamma_{0 i}(k)}
$$

Then, we formulate Lagrangian function

$$
L\left(p_{k}, \lambda\right)=-\sum_{k=2}^{n} p_{k} \ln p_{k}+\lambda\left(\sum_{k=2}^{n} p_{k}-1\right)
$$

Based on the necessary condition for the existence of extremum, we obtain

$$
\left\{\begin{array}{c}
\frac{\partial L}{\partial p_{k}}=\ln p_{k}+1-\lambda=0 \\
\frac{\partial L}{\partial \lambda}=\sum_{k=2}^{n} p_{k}-1=0
\end{array}\right.
$$

The solution is

$$
p_{k}=\frac{1}{n-1}
$$

That is to say, the distribution density values of slope incidence coefficient after weighted are equal.

As equation (3) implies

$$
\left\{\begin{array}{c}
\frac{w_{k} \gamma_{0 i}(k)}{\sum_{k=2}^{n} w_{k} \gamma_{0 i}(k)}=\frac{1}{n-1} \\
\sum_{k=2}^{n} w_{k}=1
\end{array}\right.
$$

We denote $\gamma_{0 i}(k)$ by $\gamma_{m}$, where $m=k-1$, and $k=2, \cdots, n$, then equation (8) can be expressed as

$$
\left\{\begin{array}{c}
\gamma_{1} w_{1}-\gamma_{2} w \overline{\overline{2}} 0 \\
\gamma_{2} w_{2}-\gamma_{3} w_{\overline{3}} 0 \\
\vdots \\
\gamma_{n-2} w_{n-2}-\gamma_{n} w_{-n} \overline{\overline{1}} 0 \\
\sum_{m=1}^{n-1} w_{m}=1
\end{array}\right.
$$

which can be written on matrix forms

$$
\Gamma \Omega \not
$$

where

$$
\begin{gathered}
\Gamma=\left[\begin{array}{ccccc}
\gamma_{1} & -\gamma_{2} & & \\
& \gamma_{2} & -\gamma_{3} & \\
& & \ddots & & \\
1 & 1 & 1 & 1 & 1
\end{array}\right] \\
\Omega=\left[\begin{array}{c}
w_{1} \\
w_{2} \\
\vdots \\
w_{n-2} \\
w_{n-1}
\end{array}\right], \quad b=\left[\begin{array}{c}
0 \\
0 \\
\vdots \\
0 \\
1
\end{array}\right]
\end{gathered}
$$

Then, we calculate the determinant of coefficient matrix $\Gamma$ as follows: 


$$
|\Gamma|=\left|\begin{array}{ccccc}
\gamma_{1} & -\gamma_{2} & & & \\
& \gamma_{2} & -\gamma_{3} & & \\
& & \ddots & & \\
& & & \gamma_{n-2} & -\gamma_{n-1} \\
1 & 1 & 1 & 1 & 1
\end{array}\right|
$$

For $m=3,4, \cdots, n-1$, we add the m-1-th row of $|\Gamma|$ to the $\mathrm{m}$-2-th row, the result is

$$
|\Gamma|=\left|\begin{array}{ccccc}
\gamma_{1} & & & & -\gamma_{n-1} \\
& \gamma_{2} & & & -\gamma_{n-1} \\
& & \ddots & & -\gamma_{n-1} \\
& & & \gamma_{n-2} & -\gamma_{n-1} \\
1 & 1 & 1 & 1 & 1
\end{array}\right|
$$

According to Equation (1), we know $\gamma_{m} \succ 0(m=1,2, \cdots, n-2)$.Multiply the m-th column by $\frac{\gamma_{n-1}}{\gamma_{m}}$ and then add it to the n-1-th column, it is easy to get

$$
|\Gamma|=\left|\begin{array}{rrrrr}
\gamma_{1} & & & & \\
& \gamma_{2} & & & \\
& & \ddots & & \\
& & & \gamma_{n-2} & \\
1 & 1 & 1 & 1 & +\sum_{m=1}^{n-2} \frac{\gamma_{n-1}}{\gamma_{m}}
\end{array}\right|
$$

Hence $|\Gamma|=\prod_{m=1}^{n-2} \gamma_{m}\left(1+\sum_{m=1}^{n-2} \frac{\gamma_{n-1}}{\gamma_{m}}\right)=\prod_{m=1}^{n-2} \gamma_{m}\left(\sum_{m=1}^{n-1} \frac{\gamma_{n-1}}{\gamma_{m}}\right) \succ 0 \quad$, namely, the solution of the above linear equation is existent and unique.

As a consequence, combining with equation (10), we obtain the weight vector:

$$
\Omega=\Gamma^{-1} b
$$

\section{Transformer fault diagnosis based on the model of weighted grey slope incidence}

\subsection{Establishment of normative fault mode vector for transformer}

The basic principle of grey incidence analysis applied in transformer fault diagnosis is as follows. First, the state mode vector is composed of multiple parameters which are used to characterize the state of transformer, and we establish normative fault mode vector. Then, by comparing the incidence degree between the specific state under test and the normative mode vector, we may determine which state it belongs to so that we can diagnose the transformer's fault type.

Let the gases dissolved in the transformer oil which include hydrogen $\left(\mathrm{H}_{2}\right)$ 、methane $\left(\mathrm{CH}_{4}\right)$ 、 ethylene $\left(\mathrm{C}_{2} \mathrm{H}_{4}\right)$ 、 ethane $\left(\mathrm{C}_{2} \mathrm{H}_{6}\right)$ and acetylene $\left(\mathrm{C}_{2} \mathrm{H}_{2}\right)$ be the characteristic parameters, and we establish state mode vector as follows.

$$
M=(M(1), M(2), M(3), M(4), M(5))
$$

where, $M(k)$ denotes the component content of gas.

Based on a great deal of literature reading, relative data and information collecting, normative fault mode vector of transformer has been constructed by published typical chromatographic analysis data [16-17]. Then after extensive testing and constant adjustments, we establish the normative fault mode as shown in Table 1.

Table 1. Normative mode of transformer fault $(\mu \mathrm{L} / \mathrm{L})$

\begin{tabular}{cccccc}
\hline Sequence & $\mathrm{H}_{2}$ & $\mathrm{CH}_{4}$ & $\mathrm{C}_{2} \mathrm{H}_{4}$ & $\mathrm{C}_{2} \mathrm{H}_{6}$ & $\mathrm{C}_{2} \mathrm{H}_{2}$ \\
\hline $\mathrm{M}_{1}$ & 46.1 & 21.5 & 15.8 & 61.5 & 1.2 \\
$\mathrm{M}_{2}$ & 16 & 38.4 & 28 & 70 & 0 \\
$\mathrm{M}_{3}$ & 27.5 & 48.2 & 46 & 18.4 & 0 \\
$\mathrm{M}_{4}$ & 12.95 & 24.6 & 60.6 & 12.9 & 2.8 \\
$\mathrm{M}_{5}$ & 195.9 & 14.5 & 2.4 & 11.6 & 0 \\
$\mathrm{M}_{6}$ & 61.5 & 24.6 & 5.6 & 1.33 & 20.5 \\
$\mathrm{M}_{7}$ & 75.5 & 30.2 & 30.3 & 2.33 & 18.2 \\
$\mathrm{M}_{8}$ & 4.94 & 15.8 & 3.6 & 0.88 & 14.8 \\
$\mathrm{M}_{9}$ & 14.2 & 36.4 & 30.6 & 8.5 & 24.6 \\
\hline
\end{tabular}

The normative fault mode consists of nine normative fault mode vectors, in which $\mathrm{M}_{1}$ is the sequence for normal running, $\mathrm{M}_{2}$ is the fault sequence for low temperature superheat $\left(<300^{\circ} \mathrm{C}\right), \mathrm{M}_{3}$ is the fault sequence for medium temperature superheat $\left(300 \sim 700^{\circ} \mathrm{C}\right), \mathrm{M}_{4}$ is the fault sequence for high temperature superheat $\left(>700^{\circ} \mathrm{C}\right)$, $M_{5}$ is the sequence for partial discharge, $M_{6}$ is the sequence for low-energy discharge, $\mathrm{M}_{7}$ is the sequence for 
high-energy discharge, $\mathrm{M}_{8}$ is the sequence for low-energy discharge and superheat, $\mathrm{M}_{9}$ is the sequence for high-energy discharge and superheat, so the normative fault mode vector matrix can be expressed as:

$$
M=\left[\begin{array}{c}
m_{1} \\
m_{2} \\
\vdots \\
m_{9}
\end{array}\right]=\left[\begin{array}{ccccc}
m_{1}(1) & m_{1}(2) & m_{1}(3) & m_{1}(4) & m_{1}(5) \\
m_{2}(1) & m_{2}(2) & m_{2}(3) & m_{2}(4) & m_{2}(5) \\
\vdots & \vdots & \vdots & \vdots & \vdots \\
m_{9}(1) & m_{9}(2) & m_{9}(3) & m_{9}(4) & m_{9}(5)
\end{array}\right]
$$

The fault mode vector under test is:

$$
M_{0}=\left(m_{0}(1), m_{0}(2), m_{0}(3), m_{0}(4), m_{0}(5)\right)
$$

\subsection{Case calculation}

We have collected a large amount of data of oil chromatography during transformer practical operation from an electric power company in Yunnan. First, we establish the fault mode vector under test $\left(M_{0}\right)$ for transformer, and according to equation (1), we calculate the slope incidence coefficient at each point between sequence $M_{0}$ and the sequence $M_{i}(i=1,2, \cdots, 9)$ of normative fault mode, and build the matrix $\Gamma$. Second, we calculate the weight of each point's slope by equation (14). Then, we count the weighted degree of grey slope incidence between $M_{0}$ and $M_{i}(i=1,2, \cdots, 9)$ based on equation (3).

According to the above model and method, we have done extensive experiments, and now several groups are randomly selected to prove the reliability of this method as shown in Table 2 .

Table 2. Demonstration of fault diagnosis of transformer

\begin{tabular}{ccccccccc}
\hline No. & $\mathrm{H}_{2}$ & $\mathrm{CH}_{4}$ & $\mathrm{C}_{2} \mathrm{H}_{4}$ & $\mathrm{C}_{2} \mathrm{H}_{6}$ & $\mathrm{C}_{2} \mathrm{H}_{2}$ & Actual results & $\begin{array}{c}\text { Results of the } \\
\text { improved method in } \\
\text { this paper }\end{array}$ & $\begin{array}{c}\text { Results of IEC } \\
\text { three-ratio method }\end{array}$ \\
\hline 1 & 1565 & 93 & 47 & 34 & 0 & $\begin{array}{c}\mathrm{M}_{5} \text { (partial discharge) } \\
\text { true }\end{array}$ & no coding \\
2 & 160 & 130 & 96 & 33 & 0 & $\mathrm{M}_{3}$ (medium temperature superheat) & true & false \\
3 & 56 & 78 & 173 & 18 & 0 & $\mathrm{M}_{2}$ (low temperature superheat) & false & true \\
4 & 345 & 112.3 & 51.5 & 27.5 & 58.8 & $\mathrm{M}_{6}($ low-energy discharge) & true & true \\
5 & 20.6 & 19.89 & 61.27 & 7.4 & 1.15 & $\mathrm{M}_{4}$ (high temperature superheat) & true & no coding \\
\hline
\end{tabular}

In order to test the efficiency of the method based on the weighted grey slope incidence by entropy optimization, we randomly select 300 groups data of transformer oil chromatography from the collected data,

calculate the accuracy of fault diagnosis, and then compare it with IEC three-ratio method and general grey slope incidence method. The comparison result is as shown in Table 3.

Table 3. Comparison of diagnosis result by three methods

\begin{tabular}{cccc}
\hline $\begin{array}{c}\text { diagnostic } \\
\text { method }\end{array}$ & $\begin{array}{c}\text { the modified method in } \\
\text { this paper }\end{array}$ & $\begin{array}{c}\text { IEC three-ratio } \\
\text { method }\end{array}$ & $\begin{array}{c}\text { the general grey slope-incidence } \\
\text { method }\end{array}$ \\
\hline Accuracy $/ \%$ & 92.8 & 77.5 & 89.7 \\
\hline
\end{tabular}

\section{Conclusions}

Owing to the shortage of traditional transformer fault diagnosis method, the grey incidence method is put forward to diagnose transformer's fault in this paper, and we have optimized the model of the general grey slope-incidence by entropy weight method. The extensive specific examples have proved the stability of this improved method. The new method largely make up the drawback of IEC three-ratio method, is more reasonable than the traditional ones, and effectively improve the accuracy of fault diagnosis for transformer. The diagnosis accuracy achieves $92.8 \%$ when 300 groups of random data are selected and calculated. It reduces the error caused by system interference to make the analysis results more objective.

\section{References}

1. GB/T7252-2001. Guide Anal. Diagn. Gases Di. Trans. Oil.

2. C.X. Sun, W.G. Chen, J. Li, et al. B.J.: Sci. Press, (2003).

3. J. L. Guardado, J.L. Naredo, P. Moreno, et al. IEEE Trans. Power Del., 16 ( 2001)
4. X. M. Wang, W.S. Li, Y. Zhang, High Volt. Eng., 31 (2005)

5. J. Li, C.X. Sun, W.G. Chen, et al.. Proc. Chin. Soc. Electr. Eng., 23(2003)

6. C.B. Wu. JLU, (2009)

7. Y. C. Xiao, X.M. Chen, H. G. Zhu, J.SJTU, 41(2007)

8. J. L. Deng. HUST. Press, (2002)

9. H. P. Zheng, C. X. Sun, J. Li, et al. Proc. CSEE, 21(2001)

10. S. F. Liu, Y. G. Dang, Z. G. Fang. B. J.: Sci. Press, (2004)

11. W. H. Wu. CSUST, (2009)

12. Q. S. Zhang, XJ. Guo, J.L. Deng. Syst. Eng.-Theory \& Prac., 16(1996)

13. L. Z. Cui, S.F. Liu, Z.P. Li, et al. Stat. \& Inf. Forum, 25(2010)

14. P. Purkait, S. Chakravorti. DEI, 9 (2002)

15. B. Song, P. Yu, Y.B. Luo, et al. Power Syst. Technol. Int. Conf. Power Con. K.M., (2002)

16. H. G. Deng, A. Luo, J. Cao, et al. Autom. Electr. Power Syst., 29(2005)

17. M. Dong, Z. Yan, L. Yang, et al. Proc.-Chin. Soc. Electr. Eng., 26(2006) 